\title{
Development of multivariable models to predict perinatal depression before and after delivery using patient reported survey responses at weeks 4-10 of pregnancy
}

Jenna Reps ( $\sim$ jreps@its.jnj.com )

Janssen Research \& Development https://orcid.org/0000-0002-2970-0778

Marsha Wilcox

Janssen Research and Development Titusville

Beth Ann McGee

BabyCenter

Marie Leonte

BabyCenter

Lauren Lacross

BabyCenter

Kevin Wildenhaus

Janssen Research and Development Titusville

Research article

Keywords: patient-level prediction, perinatal depression, machine learning, model development

Posted Date: July 30th, 2021

DOI: https://doi.org/10.21203/rs.3.rs-757801/v1

License: (c) (i) This work is licensed under a Creative Commons Attribution 4.0 International License. Read Full License 


\section{Abstract \\ Background}

Perinatal depression is estimated to affect $\sim 12 \%$ of pregnancies and is linked to numerous negative outcomes. There is currently no model to predict perinatal depression at multiple time-points during and after pregnancy.

\section{Methods}

A prospective cohort design where 858 participants filled in a baseline self-reported survey at week 4-10 of pregnancy (that included social economics, health history, various psychiatric measures), with follow-up until 3 months after delivery. Our primary outcome was an Edinburgh Postnatal Depression Score (EPDS) score of 12 or more (a proxy for perinatal depression) assessed during each trimester and again at two time periods after delivery. Five gradient boosting machines were trained to predict the risk of having EPDS score $>=12$ at each of the five follow-up periods. We investigated different predictor sets.

\section{Results}

We developed five prognostic models: PND-T1 (trimester 1), PND-T2 (trimester 2), PND-T3 (trimester 3), PND-A1 (after delivery 1) and PND-A2 (delayed onset after delivery) that calculate personalised risks while only requiring that women be asked 21 questions from 3 validated psychometric scales at weeks 4-10 of pregnancy. C-statistics ranged between $0.69(0.65-0.73)$ and $0.77(0.74-0.80)$. At $50 \%$ sensitivity the precision ranged between $30 \%-50 \%$ across the models, generally identifying groups of patients with double the average risk.

\section{Conclusions}

The five models can predict risk of perinatal depression within each trimester and in two post-natal periods using survey responses as early as week 4 of pregnancy. The models need to be externally validated and prospectively tested to ensure generalizability to any pregnant patient.

\section{Background}

Perinatal depression (PND) is depression that occurs during or shortly after pregnancy and research has shown it to be a cause of numerous negative outcomes for the affected women's children (Grote 2010; Alder 2007; Murray 1997). It is estimated to impact approximately $12 \%$ of pregnant women (Woody 2017 ) and there is a lack of methodology to predict those at risk in the general population (Alder 2007). If predictions were available that could identify the higher risk patient population, then potential interventions could be developed to reduce this number through prevention and early interception of PND and improve health and wellbeing outcomes for both pregnant women and their children.

Researchers have identified numerous risk factors of perinatal or postpartum depression, including various psychological factors such as mental health issues prior to pregnancy (Patton 2015), state and trait anxiety (Enatescu 2014), poor relationships, stressful events and negative attitudes towards pregnancy (Norhayati 2015). The type of delivery such as emergency caesarean has also been linked to postpartum depression (Boyce 1992) as well as social support (Norhayati 2015) and being a housewife (Goker 2012). Although many predictors have been identified, there is a lack of clinically useful predictive models that can be applied during the early stage of pregnancy to identify women at high risk for PND. 
Examples of published models that predict postpartum depression include a logistic regression using antenatal variables such as age, marital status, occupational status, history of psychiatric disease, perceived social isolation and psychological distress during pregnancy was able to predict postpartum depression 4 month postpartum with a positive predictive value of $30 \%$, sensitivity of $79 \%$ and specificity of $50 \%$ (Nielsen 2000 ). Another study developed a logistic regression model to predict postpartum depression 6-8 weeks postpartum using 17 variables self-reported in the third trimester and obtained a sensitivity of $33 \%$, specificity of $87 \%$ and positive predictive value of $35 \%$ (Cooper 1996). The Brisbane Postnatal Depression Index included antenatal and postnatal variables to predict postpartum depression (16 weeks postpartum) and obtained slightly better performance with a $36.3 \%$ sensitivity, $92 \%$ specificity and a $40 \%$ positive predictive value (Webster 2003). These models generally require variables collected during pregnancy, limiting their application to later stages of the pregnancy. In addition, they tend to focus on predicting depression after delivery. However, a recent study identified $80 \%$ onset in pregnancy, further highlighting the importance of early identification of risk (Wilcox 2021).

In this paper we aim to develop clinically useful models that can predict depression during each trimester (weeks 12/13, 21 and 32) and at weeks 4 and 12 post-delivery using variables that are ascertained in weeks 4-10 of pregnancy.

\section{Materials \& Methods}

\section{Prediction Questions}

The prediction question answered in this paper is:

Within pregnant women, predict a self-reported EPDS of 12 or more (proxy for PND) at weeks 12/13, 21, 32 of pregnancy and weeks 4 and 12 post-delivery using the baseline (week 4-10 of pregnancy) survey responses as predictors.

\section{Source of Data}

This study was a prospective study containing self-reporting survey data measured at multiple time points during and after pregnancy. The survey was advertised to women interacting with the BabyCenter website, an educational and informational website for moms, spouses and partners. Women were enrolled into the study between week 4 to week 10 of their pregnancy. The participants filled in a survey asking them about their lifestyle, social economics, health history and five psychiatric measures at enrolment (baseline). The participants were then followed longitudinally throughout pregnancy and after delivery and the Edinburgh Postnatal Depression Score (EPDS) survey was given to participants five times after baseline (during each trimester and two times after delivery), see Fig. 1. The first EPDS score post baseline used in this study was given at weeks 12 or 13 depending on the baseline enrolment week. This was administered between 3 weeks and 8 weeks after baseline. Two more EPDS scores during pregnancy (trimester 2 at week 21 and trimester 3 at week 32) and two more EPDS scores after delivery (week 4 and week 12 post-delivery) were also included in this study.

\section{Participants}

The participants of this study were women who were active on the BabyCenter website, between August 25 to September 19,2016 , and consented for participation. Eligibility criteria were that the participant must be 4-10 weeks pregnant at enrolment. Participants were excluded if any of the following were true: male gender, location outside the US, age less than 18 , or participating in other studies. The sample of pregnant women enrolled into the study appear to be representative of the US adult population (McGee 2021). 
Participants were paid for each survey completed and could have received up to $\$ 180$ if all surveys were completed. In addition, participants were included into a $\$ 1000$ sweepstake and the number of entries per person depended on how many surveys they completed.

\section{Outcome}

We predicted perinatal depression during five different time points: each trimester and at two periods after delivery. Perinatal depression at a given time period was defined as an EPDS score (the most recent during the time period) of 12 or more.

The EPDS is a measure that has been developed to assess the risk of perinatal depression but is not a clinical diagnosis of depression. An EPDS of 14 or more is often used as a cut off to divide into high risk and low risk of depressive illness. In this study we used an EPDS score of 12 or more as a proxy for depression as the self-harm question was missing from the self-reported survey used in this study due to ethical considerations. It has been shown that the EPDS score identified major depression with a sensitivity of $88 \%$, a specificity of $92.5 \%$ and a positive predictive value of $35.1 \%$ (Murray 1990).

\section{Predictors}

We used the self-reported baseline survey response to construct predictors. The baseline survey included 180 different questions on lifestyle, social economics, health history, various psychiatric measures (e.g., state-trait anxiety inventory, generalized anxiety disorder (GAD) (Spitzer 2006), PROMIS emotional support (PRES) (PROMIS 2016) and perceived stress scale (PSS) (Cohen 1983)) and the baseline EPDS questions (excluding the self-harm question). The generalized anxiety disorder scale contains 8 questions, the EPDS (less the suicide question) contains 9 questions and the PROMIS emotional support contains 4 questions.

The baseline predictors are a combination or ordinal variables, binary indicator variables and category variables. Every participant filled out the baseline survey, but some questions were optional.

\section{Sample Size}

5,028 BabyCenter users showed interest in completing the survey. 3,471 were excluded due to pregnancy outside weeks of interest $(2,186)$, not completing the screening Sect. (557), not being pregnant (317), participating in other research (190), age less than 18 (151), located outside the US (75) and not being male (55). This left 1,557 qualified to participate and 1,179 (76\%) completed the baseline survey. Eight hundred and fifty-eight (858) of these participants were 4-10 weeks pregnant and 321 were 28 to 33 weeks pregnant. This study only used the 858 participants at 4-10 weeks of their pregnancy who completed the baseline survey. 554, 528, 555, 469 and 515 of these participants filled in the EPDS survey during trimester 1 (week 12 or 13), trimester 2 (week 21), trimester 3 (week 32), week 4 post-delivery and week 12 postdelivery, respectively.

\section{Missing Data}

Predictors: All participants filled out the baseline survey, but some questions were optional resulting in some missing data. To address this, we excluded 16 baseline variables due to insufficient responses (a binary indicator where nobody selected 'Yes' or an ordinal/category response that was answered by $<50 \%$ of participants). For the remaining ordinal and category variables we used mode imputation when values were missing. For the indicator variables, we only had 'Yes' responses from participants, so we imputed 'No' when a 'Yes' was not selected.

Outcome: The follow-up EPDS surveys were not compulsory and were missed by a significant number of participants. We excluded patients from the data used to train and evaluate each model if they did not complete the EPDS survey at the specific time point being predicted. We investigated the differences in baseline responses between patients who were 
excluded and those used in each model development to quantify how excluding patients without the outcome may compromise generalizability of the models.

\section{Statistical Analysis Methods}

We investigated three different predictor sets:

1. [GAD/EPDS] Baseline generalized anxiety disorder and EPDS scale questions

2. [GAD/EPDS/PRES] Baseline generalized anxiety disorder, EPDS and the PROMIS emotional support scale questions

3. [All 164 predictors] Baseline non-scale questions (e.g., health history, demographics, lifestyle, partner's mental health) plus the generalized anxiety disorder, EPDS and the PROMIS emotional support questions

For each outcome and predictor set we trained a gradient boosting machine (Friedman 2001). We split the data into $80 \%$ training and $20 \%$ testing sets. We used 10 -fold cross validation repeated ten times on the training data to identify the optimal hyper-parameters and then trained a final model with the optimal hyper-parameters using all the training data. We internally evaluated the model on the $20 \%$ test set by calculating the discriminative ability using the area under the receiver operating characteristic curve (AUROC). The sensitivity (percentage of the actual depressed patients that are predicted to be depressed) and positive predictive values (the percentage of actual depressed people in the patients predicted to be depressed) are also presented at various thresholds. We repeated the above process 10 times with different train/test splits to calculate confidence intervals for the performance estimates.

To calculate the predictor importance, we used SHapley Additive explanation (SHAP) (Lundberg 2017). SHAP uses a game theory approach to estimate the impact that each predictor has on participants' predicted risks. This can provide predictor important globally across all participants as well as locally for a specific participant.

\section{Results}

\section{Participants}

858 women were enrolled into the study and filled in the baseline survey. 554, 528, 555, 469 and 515 of these women filled in the EPDS survey during trimester 1, trimester 2, trimester 3, week 4 post-delivery and week 12 post-delivery, respectively. To quantify whether the women who completed the EPDS survey at each follow-up time-period were different at baseline from those who did not, we developed models to predict who would participate in each follow-up EPDS survey using all baseline predictors and 10-fold cross validation repeated 10 times. The ability to predict participation was moderately weak with cross-validation AUROCs ranging between $0.63-0.66$ across the time periods. This suggests there are some small baseline differences in those who drop out and those who do not. Some of the baseline responses associated with participation were being married, ethnicity, not feeling upset at baseline, using a desktop computer, history of yoga, partner not having any existing mental health issues, diet, sleep issues and income.

The full details of the characteristics of the missing vs present people at each follow-up timepoint are presented in Supplement A.

\section{Gradient boosting machine models}

The target population and outcome sizes are presented in Table 1. 
Table 1

The data sizes and outcome count for the different time-periods investigated

\begin{tabular}{|llll|}
\hline Follow-up Period & Target Population & Outcome Count & Outcome \% \\
\hline Trimester 1 & 554 & 116 & 20.9 \\
\hline Trimester 2 & 528 & 111 & 20.0 \\
\hline Trimester 3 & 555 & 140 & 25.2 \\
\hline After deliver 1 & 469 & 77 & 16.4 \\
\hline After deliver 2 & 515 & 89 & 17.3 \\
\hline
\end{tabular}

The baseline characteristics of those who have an EPDS score of 12 or greater during each time period and those who do not are presented in supplement $B$. The performance of the models for each EPDS follow-up time period and predictor set are presented in Table 2. Receiver Operating Characteristic (ROC) curves and calibration plots for the models using the $21 \mathrm{GAD} / E P D S / P R E S$ predictors are presented in Figs. 1 and 2.

Table 2

The discriminative performance of the models using different predictor sets

\begin{tabular}{|c|c|c|c|c|c|c|}
\hline \multirow[b]{2}{*}{ Predictor Set } & \multirow[b]{2}{*}{$\begin{array}{l}\text { Predictor } \\
\text { Count }\end{array}$} & \multicolumn{5}{|c|}{ AUROC (95\% Cl) } \\
\hline & & Trimester 1 & Trimester 2 & Trimester 3 & $\begin{array}{l}\text { After delivery } \\
1\end{array}$ & $\begin{array}{l}\text { After delivery } \\
2\end{array}$ \\
\hline GAD/EPDS & 17 & $\begin{array}{l}0.78(0.75- \\
0.80)\end{array}$ & $\begin{array}{l}0.69(0.65- \\
0.74)\end{array}$ & $\begin{array}{l}0.73(0.69- \\
0.78)\end{array}$ & $\begin{array}{l}0.71(0.67- \\
0.76)\end{array}$ & $\begin{array}{l}0.69(0.64- \\
0.73)\end{array}$ \\
\hline GAD/EPDS/PRES & 21 & $\begin{array}{l}0.77(0.74- \\
0.80)\end{array}$ & $\begin{array}{l}0.69 \\
0.73)\end{array}$ & $\begin{array}{l}0.75(0.71- \\
0.79)\end{array}$ & $\begin{array}{l}0.72(0.67- \\
0.78)\end{array}$ & $\begin{array}{l}0.71(0.66- \\
0.76)\end{array}$ \\
\hline All Predictors & 164 & $\begin{array}{l}0.75(0.72- \\
0.78)\end{array}$ & $\begin{array}{l}0.69(0.65- \\
0.72)\end{array}$ & $\begin{array}{l}0.74(0.71- \\
0.78)\end{array}$ & $\begin{array}{l}0.73(0.68- \\
0.77)\end{array}$ & $\begin{array}{l}0.70(0.64- \\
0.77)\end{array}$ \\
\hline
\end{tabular}

The SHAP variable importance for each model using the GAD/EPDS/PRES predictor set is presented in Supplement C.

The positive predictive value (PPV) of the GAD/EPDS/PRES models at various sensitivity cut points are presented in Table 3. 
Table 3

The sensitivity, positive predictive value (PPV) at various risk threshold for the five models using the EPDS/GAD/PSS predictors

\begin{tabular}{|lllllllllll|l|}
\hline Sensitivity & Trimester 1 & \multicolumn{3}{c}{ Trimester 2 } & \multicolumn{3}{c}{ Trimester 3 } & \multicolumn{3}{c|}{ After delivery 1 } & \multicolumn{2}{c|}{ After delivery 2 } \\
\hline & Threshold & PPV & Threshold & PPV & Threshold & PPV & Threshold & PPV & Threshold & PPV \\
\hline $10 \%$ & 0.80 & 75.0 & 0.65 & 52.4 & 0.70 & 60.9 & 0.54 & 33.3 & 0.58 & 45.0 \\
\hline $20 \%$ & 0.66 & 67.6 & 0.54 & 50.0 & 0.60 & 59.6 & 0.45 & 40.5 & 0.42 & 37.5 \\
\hline $30 \%$ & 0.52 & 61.4 & 0.43 & 46.5 & 0.51 & 57.5 & 0.39 & 42.6 & 0.33 & 35.5 \\
\hline $40 \%$ & 0.44 & 60.5 & 0.34 & 43.1 & 0.43 & 53.8 & 0.30 & 36.0 & 0.26 & 31.3 \\
\hline $50 \%$ & 0.32 & 49.2 & 0.27 & 41.1 & 0.37 & 49.3 & 0.25 & 34.5 & 0.21 & 29.5 \\
\hline $60 \%$ & 0.25 & 45.2 & 0.21 & 35.6 & 0.30 & 46.2 & 0.21 & 31.9 & 0.17 & 28.5 \\
\hline $70 \%$ & 0.18 & 40.5 & 0.15 & 32.0 & 0.22 & 40.8 & 0.17 & 30.2 & 0.15 & 29.0 \\
\hline $80 \%$ & 0.13 & 33.7 & 0.12 & 29.8 & 0.14 & 36.6 & 0.12 & 27.9 & 0.11 & 27.1 \\
\hline $90 \%$ & 0.07 & 27.2 & 0.08 & 26.2 & 0.10 & 33.2 & 0.07 & 24.8 & 0.07 & 23.5 \\
\hline
\end{tabular}

\section{Discussion \& Conclusion}

\section{Interpretation}

We investigated models that predict depression (using EPDS $>=12$ as a proxy) at five time periods during and after pregnancy using survey responses during weeks 4-10 of pregnancy. In the first two trimesters approximately $20 \%$ of the women surveyed had an EPDS > = 12, this increased to $25 \%$ in the final trimester and then decreased to $16 \%-17 \%$ following delivery.

We developed models using three predictor sets: i) 17 questions from the EPDS and GAD scales, ii) 21 questions from the EPDS, GAD and PRES scales and iii) 164 questions including additional psychiatric scales, demographics, lifestyle, medical history and partner mental health questions. The results show that the performance was similar for all three predictor sets, with models tending to overfit when all 164 variables were used due to the small data size. Including the PRES scale questions tended to improve the prediction of depression after delivery, although the performance was not significantly better than the models using EPDS/GAD only.

The SHAP results show that crying early in pregnancy is a key predictor of high EPDS scores during pregnancy. In general, showing signs of depression/anxiety at week 4-10 was predictive of a high EPDS throughout pregnancy. Baseline predictors of a high EPDS after delivery were anxiety (worrying, nervousness and anxiety), difficulty sleeping and feeling afraid. Having somebody who makes you feel appreciated appears to be associated with lower EPDS score after pregnancy, however causality was not investigated in this study.

Focusing on the models developed using the 21 questions from the EPDS, GAD and PRES scales, the models AUROC performance across the time periods ranged between low to middle 70s, with trimester 1 being the easier to predict. This is expected, as trimester 1 was closest in time to the baseline survey. The calibration plots indicate reasonable calibration, although the models appear to slightly over-estimate risk for the highest risk groups. When predicting an EPDS > = 12 after delivery during weeks 4 and 12, the calibration plots show there is a group of women who are assigned a risk around $10 \%$ but approximately $25 \%$ of these women had an EPDS $>=12$. This may be due to the model using variables early in pregnancy, which may be insufficient to identify these women as high risk after delivery. 
To use the models a patient would need to be asked only 21 questions at week 4 to 10 of their pregnancy. These 21 items could easily be assessed via online survey, phone or tablet to determine risk at the different time-points. If using the models for decision making, we provide the positive predictive values (PPV) and sensitivities for ten different thresholds, see Table 5. The desirable threshold will depend on how the models will be used. For example, if the model is used to simply screen patients who may benefit from additional education, then a high sensitivity may be preferred at the cost of having a higher false positive rate (lower PPV). Alternatively, if the models are used to identify patients who may benefit from some restricted intervention, then a high PPV may be more desirable.

\section{Implications}

Our models can be implemented early in pregnancy (week 4-10) by asking women to complete 3 common psychological scales to calculate a personal risk of developing depression at different time points during and after delivery. If a patient is assigned into the higher risk groups, then the care provider may wish to educate the patient more about perinatal depression and the symptoms or set up more regular appointments.

\section{Limitations}

The main limitation of this study is that a high percentage of women dropped out after the baseline survey. This may impact the generalizability of the models to the general population as the dropout may be associated with having or developing depression. We were unable to find any strong predictor of dropout using baseline variables. This suggests that the model may be generalizable, however it is import for these models to be externally validated to confirm this. Based on GRASP guidelines, the models need to be externally validated and also prospectively tested in any clinically setting they may be applied before the true performance is known (Khalifa 2019). Another limitation is that the EPDS score was used as a proxy for depression and the EPDS score is not a clinical diagnosis. In future work it would be useful to validate the model on data that has a clinical definition of perinatal depression as the outcome.

As we used gradient boosting machines, the models are hard to interpret. We used SHAP to provide variable importance plots to show which variables had more impact in the risk predictions. SHAP can also be used when using the online calculator to understand what contributed to the high risk.

A key strength of this study is that it used a prospective cohort design, but this resulted in having a smaller dataset of around 500-600 patients and outcome counts ranging between 77-140. The low outcome count limited the complexity of the models, so more discriminative models may be possible to learn with more data. It also decreases the confidence in the model performance estimates, leading to wider confidence intervals.

\section{Conclusion}

In this paper we developed five models that only require asking 21 questions at week 4-10 of pregnancy and can be used to predict whether a patient is at high or low risk of experiencing depression during each trimester and during two time periods after delivery. The models could be used to identify patients who would benefit from certain interventions, such as additional education about depression or more regular check-ups. In future work it is important to examine the generalizability of the models by externally validating them on new patients. It would also be beneficial to test the models' performances when using clinically defined depression as the outcome.

\section{Declarations}

Ethics approval and consent to participate All procedures performed in studies involving human participants were in accordance with the ethical standards of the institutional and/or national research committee and with the $1964 \mathrm{Helsinki}$ 
declaration and its later amendments or comparable ethical standards. This study received New England IRB approval (now called WCG IRB) and written informed consent was obtained from all individual participants included in the study.

\section{Consent to publication Not applicable}

Availability of data and material The data that support the findings cannot be shared due to privacy/ethical restrictions

Competing interests All authors are employees of Johnson \& Johnson. Baby Center was a Johnson \& Johnson entity at the time this work was done.

Funding This study was funded by Janssen Research \& Development, LLC, a Johnson \& Johnson company.

Code availability This study was conducted using the open-source programming language R using the caret package.

Authors' contributions JMR, MW and KW designed the study and JMR lead the data analysis. BAM, ML, LL and KW were involved in the data collection. All authors interpreted the results. All authors read and approved the final manuscript

Acknowledgements Not Applicable

\section{References}

1. Alder, J., Fink, N., Bitzer, J., Hösli, I. and Holzgreve, W., 2007. Depression and anxiety during pregnancy: a risk factor for obstetric, fetal and neonatal outcome? A critical review of the literature. The Journal of Maternal-Fetal \& Neonatal Medicine, 20(3), pp.189-209.

2. Boyce, P.M. and Todd, A.L., 1992. Increased risk of postnatal depression after emergency. Med. J. Australia, 157(3), pp.172-174.

3. Cohen, S., Kamarck, T., and Mermelstein, R., 1983. A global measure of perceived stress. Journal of Health and Social Behavior, 24, pp.386-396.

4. Cooper, P.J., Murray, L., Hooper, R. and West, A., 1996. The development and validation of a predictive index for postpartum depression. Psychological medicine, 26(3), pp.627-634.

5. Enatescu, V.R., Enatescu, I., Craina, M., Gluhovschi, A., Papava, I., Romosan, R., Marian, C., Oprea, A. and Bernad, E., 2014. State and trait anxiety as a psychopathological phenomenon correlated with postpartum depression in a Romanian sample: a pilot study. Journal of Psychosomatic Obstetrics \& Gynecology, 35(2), pp.55-61.

6. Friedman, J.H., 2001. Greedy function approximation: a gradient boosting machine. Annals of statistics, pp.11891232.

7. Goker, A., Yanikkerem, E., Demet, M.M., Dikayak, S., Yildirim, Y. and Koyuncu, F.M., 2012. Postpartum depression: is mode of delivery a risk factor?. ISRN obstetrics and gynecology, 2012.

8. Grote, N.K., Bridge, J.A., Gavin, A.R., Melville, J.L., lyengar, S. and Katon, W.J., 2010. A meta-analysis of depression during pregnancy and the risk of preterm birth, low birth weight, and intrauterine growth restriction. Archives of general psychiatry, 67(10), pp.1012-1024.

9. Khalifa, M., Magrabi, F. \& Gallego, B., 2019. Developing a framework for evidence-based grading and assessment of predictive tools for clinical decision support. BMC Med Inform Decis Mak, 19(1), pp.1-17

10. Lundberg, S.M. and Lee, S.I., 2017. A unified approach to interpreting model predictions. In Advances in neural information processing systems, pp. 4765-4774.

11. McGee B, Leonte M, Wildenhaus K, Wilcox M, Reps J, LaCross L, 2021. Leveraging Digital Technology in Conducting Longitudinal Research on Mental Health in Pregnancy: Longitudinal Panel Survey Study. JMIR Pediatr Parent, 4(2):e16280 
12. Murray, L. and Carothers, A.D., 1990. The validation of the Edinburgh Post-natal Depression Scale on a community sample. The British Journal of Psychiatry, 157(2), pp.288-290.

13. Murray, L. and Cooper, P.J., 1997. Effects of postnatal depression on infant development. Archives of disease in childhood, 77(2), pp.99-101.

14. Nielsen, D., Videbech, P., Hedegaard, M., Dalby, J. and Secher, N.J., 2000. Postpartum depression: identification of women at risk. BJOG: An International Journal of Obstetrics \& Gynaecology, 107(10), pp.1210-1217.

15. Norhayati, M.N., Hazlina, N.N., Asrenee, A.R. and Emilin, W.W., 2015. Magnitude and risk factors for postpartum symptoms: a literature review. Journal of affective Disorders, 175, pp.34-52.

16. Patton, G.C., Romaniuk, H., Spry, E., Coffey, C., Olsson, C., Doyle, L.W., Oats, J., Hearps, S., Carlin, J.B. and Brown, S., 2015. Prediction of perinatal depression from adolescence and before conception (VIHCS): 20-year prospective cohort study. The Lancet, 386(9996), pp.875-883.

17. PROMIS. (2016). PROMIS Item Bank v2.0, Emotional Support Short Form 4a. Retrieved from http://www.healthmeasures.net/administrator/components/com_instruments/uploads/PROMIS SF v2.0 Emotional Support 4a 6-23-2016.pdf [Google Scholar]

18. Spitzer, R.L., Kroenke, K., Williams, J.B. and Löwe, B., 2006. A brief measure for assessing generalized anxiety disorder: the GAD-7. Archives of internal medicine, 166(10), pp.1092-1097.

19. Webster, J., Pritchard, M.A., Creedy, D. and East, C., 2003. A simplified predictive index for the detection of women at risk for postnatal depression. Birth, 30(2), pp.101-108.

20. Woody, C.A., Ferrari, A.J., Siskind, D.J., Whiteford, H.A. and Harris, M.G., 2017. A systematic review and metaregression of the prevalence and incidence of perinatal depression. Journal of affective disorders, 219, pp.86-92.

21. Wilcox, M., McGee, B., Ionescu, D.F. et al., 2021. Perinatal depressive symptoms often start in the prenatal rather than postpartum period: results from a longitudinal study. Arch Womens Ment Health, 24, pp.119-131.

\section{Figures}

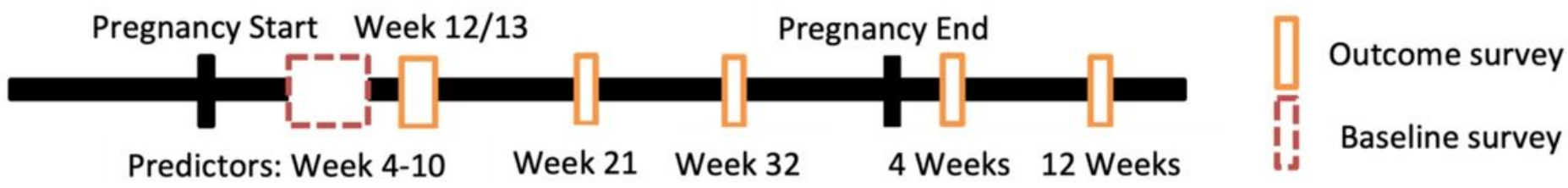

Figure 1

Survey timeline used to capture the data for this study 

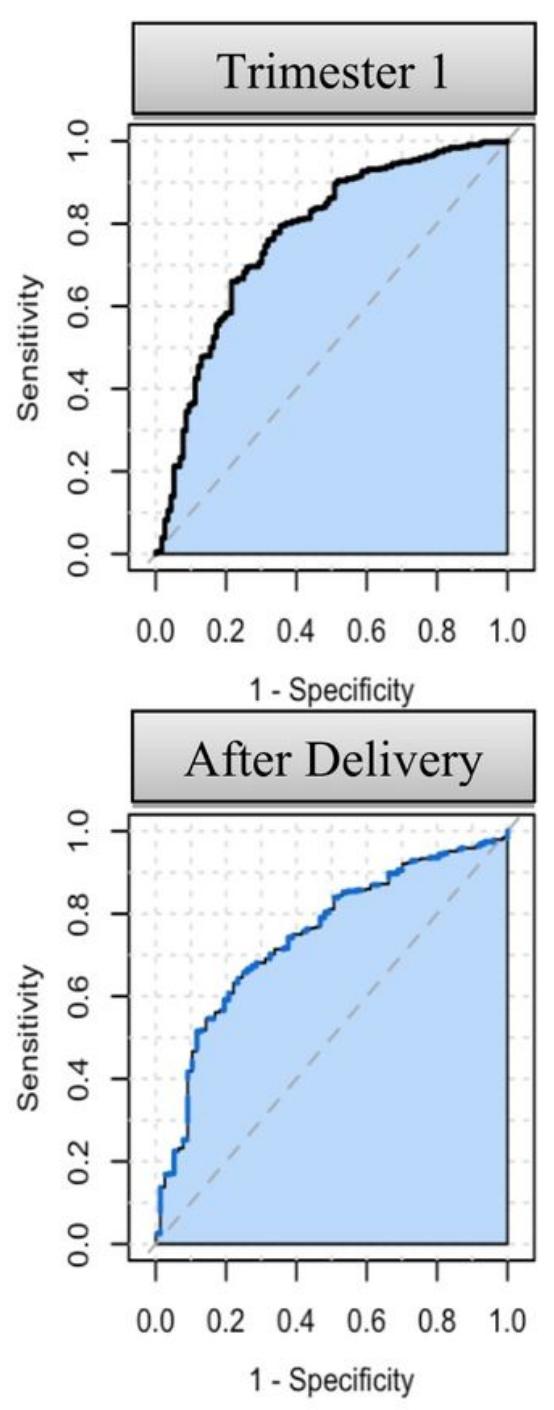
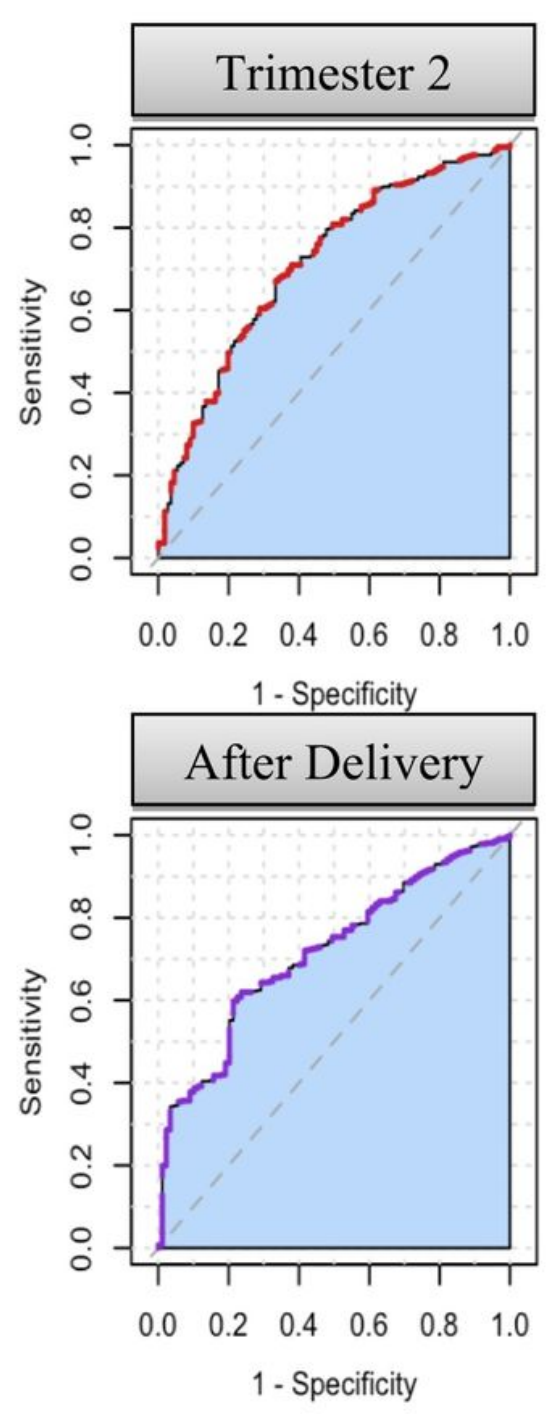

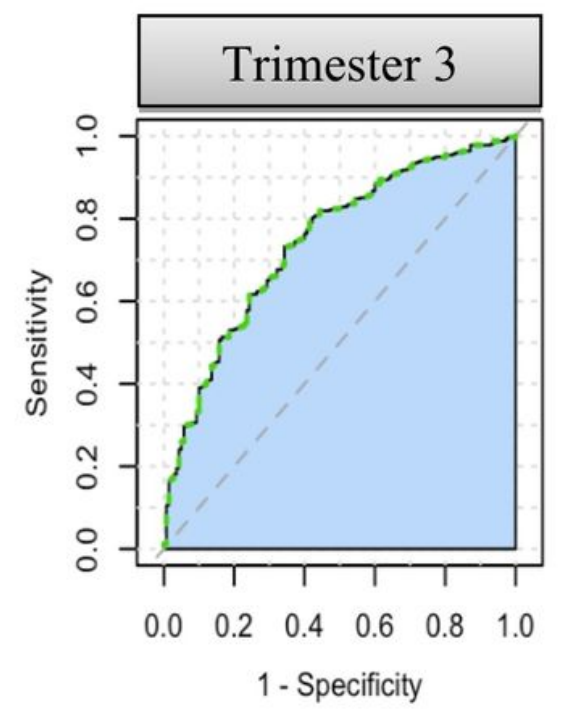

Figure 2

ROC plots for the five GBM models using EDPS/GAD/PRES predictors 


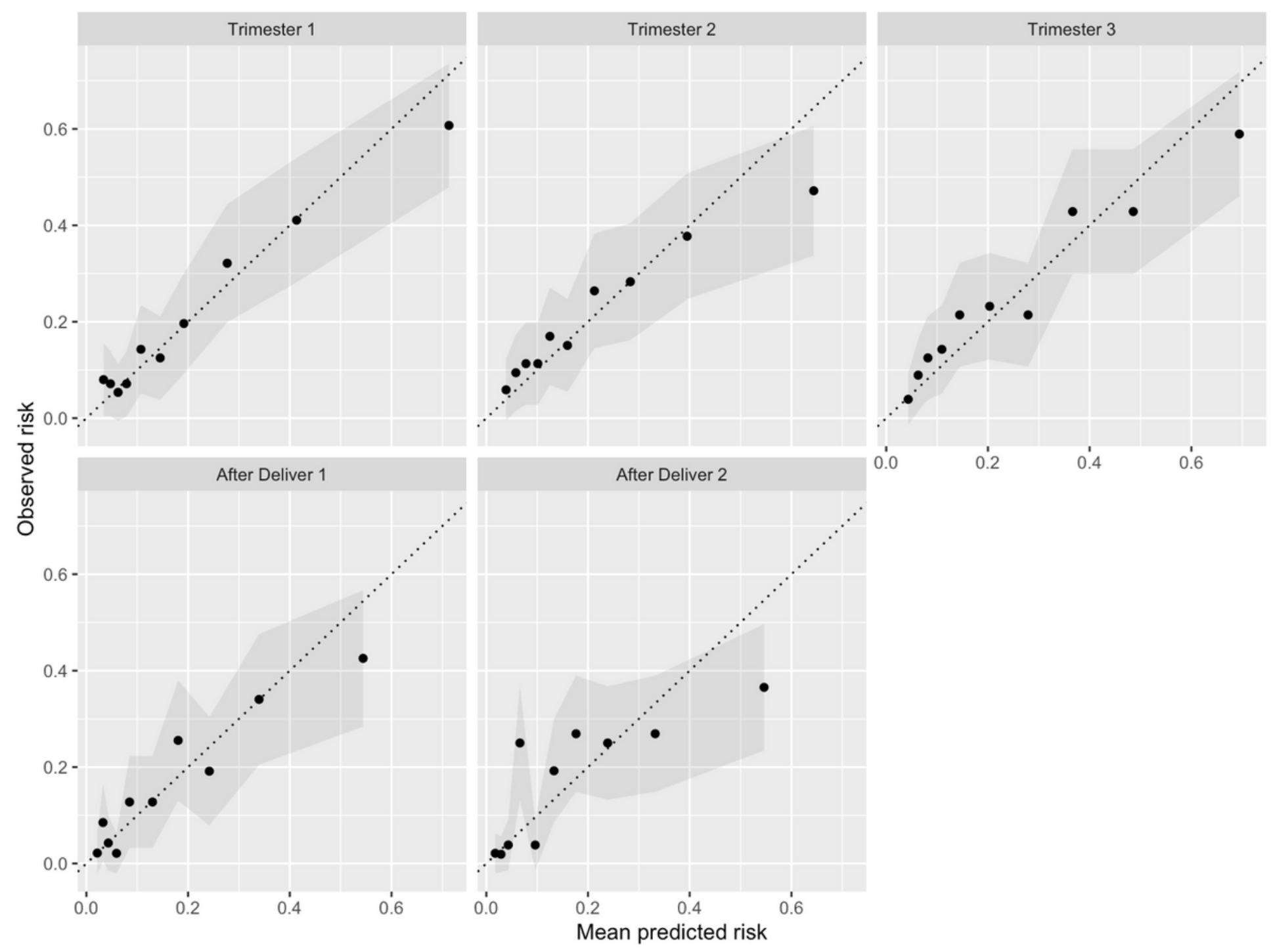

Figure 3

Calibration plots for the five GBM models using EPDS/GAD/PRES predictors

\section{Supplementary Files}

This is a list of supplementary files associated with this preprint. Click to download.

- SupplementA.docx

- SupplementB.docx

- SupplementC.docx 